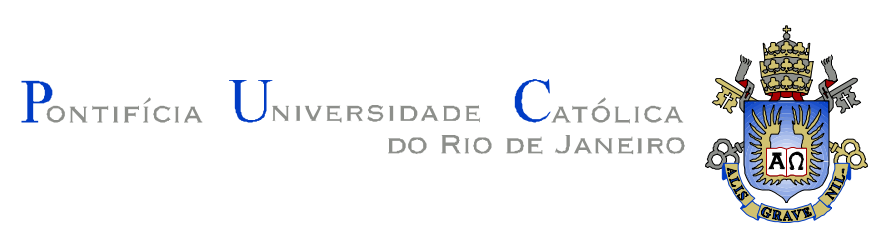

Giancarlo Vilela de Faria

\title{
Controle da polarização da luz em fibras ópticas monomodo e aplicações
}

\begin{abstract}
Tese de Doutorado
Tese apresentada ao Programa de Pós-Graduação em Engenharia Elétrica como requisito parcial para obtenção do título de Doutor em Engenharia Elétrica.

Orientador: Jean Pierre von der Weid
\end{abstract}

Rio de Janeiro, Março de 2009 


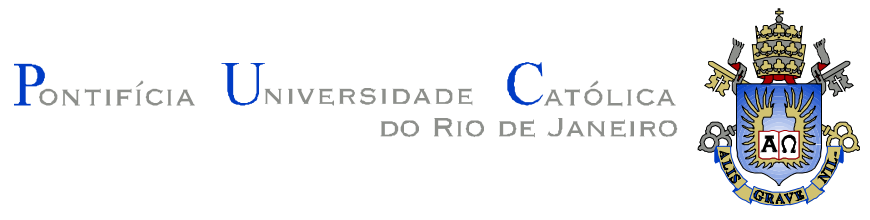

Giancarlo Vilela de Faria

\section{Controle da polarização da luz em fibras ópticas monomodo e aplicações}

Tese apresentada como requisito parcial para obtenção do título de Doutor pelo Programa de Pós-Graduação em Engenharia Elétrica do Departamento de Engenharia Elétrica do Centro Técnico Científico da PUC-Rio. Aprovada pela Comissão Examinadora abaixo assinada.

Prof. Jean Pierre von der Weid Orientador Centro de Estudos em Telecomunicações - PUC-Rio

Prof. Rogério Passy MLS Wireless

Prof. Alexandre de Oliveira Dal Forno UERJ - Intelig

Prof. Marcelo Roberto Baptista Pereira Luis Jimenez

Centro de Estudos em Telecomunicações - PUC-Rio

Prof. Guilherme Penello Temporão Centro de Estudos em Telecomunicações - PUC-Rio

Prof. Luis Carlos Blanco Linares Lupatech Monitoring Systems

Prof. Djeisson Hoffmann Thomas Centro de Estudos em Telecomunicações - PUC-Rio

Prof. José Eugênio Leal Coordenador(a) Setorial do Centro Técnico Científico - PUC-Rio

Rio de Janeiro, 27 de Março de 2009 
Todos os direitos reservados. É proibida a reprodução total ou parcial do trabalho sem autorização da universidade, do autor e do orientador.

\section{Giancarlo Vilela de Faria}

Graduou-se em Engenharia Elétrica (Eletrônica), em 2002, na Pontifícia Universidade Católica do Rio de Janeiro. No mesmo ano, fixou-se como engenheiro no Centro de Estudos em telecomunicações da Pontifícia Universidade Católica do Rio de Janeiro. Em 2003 iniciou seu mestrado, o qual, concluiu em 2005 e iniciou o doutorado desenvolvendo pesquisas na área de comunicações ópticas, metrologia óptica e optoeletrônica.

Ficha Catalográfica

Faria, Giancarlo Vilela de
Controle da polarização da luz em fibras ópticas
monomodo e aplicações / Giancarlo Vilela de Faria ;
orientador: Jean Pierre Von der Weid. - 2009.
102 f. : il.(col.) ; $29,7 \mathrm{~cm}$
Tese (Doutorado em Engenharia Elétrica)-
Pontifícia Universidade Católica do Rio de Janeiro, Rio de
Janeiro, 2009.
Inclui bibliografia
1. Engenharia elétrica - Teses. 2. Polarização.
2. Controle da polarização. 3. Dispersão dos modos de
polarização. 4. Comunicações quânticas. I. Weid, Jean
Pierre Von der. II. Pontifícia Universidade Católica do Rio
de Janeiro. Departamento de Engenharia Elétrica. III.
Título.




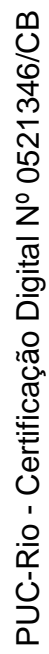

"A essência do conhecimento consiste em aplicá-lo, uma vez possuído."

\section{Confúcio}




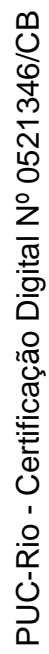

À minha esposa Gabriela e aos meus pais Haroldo e Rita. 


\section{Agradecimentos}

Ao prof. Jean Pierre, pela orientação, incentivo, oportunidade, confiança e amizade ao longo de todos esses anos.

À minha querida esposa Gabriela, pela enorme paciência, carinho e pelo importante companheirismo de todos os dias.

Aos meus pais Haroldo e Rita, pelo incentivo, amor, educação e principalmente pelos seus exemplos.

Aos meus irmãos e suas famílias pelo apoio e presteza nos momentos difíceis.

Ao pessoal do laboratório, Andy, Djeisson, Janaina, Karoline, Thiago pelo companheirismo, e em especial ao Guilherme (Guix) e Temporão, pelas contribuições e discussões.

Ao pessoal do CPTI pelo suporte em geral, em especial ao Marcelo Roberto pelo desenvolvimento do software para o primeiro modelo do compensador.

Aos estagiários, Fernando, Gustavo e em especial ao Rogério (Chu) e Tarcísio, pelo grande auxilio no desenvolvimento do protótipo final do sistema utilizado.

À Amália e Mônica, pelo suporte burocrático.

Aos meus sogros, Celinho e Palmirão, e meus cunhados pela compreensão, confiança e apoio. 
À Evely Martins e Marco Câmera, pelo auxilio com os experimentos nos laboratórios da Ericsson em Gênova.

Ao Nino Walenta, Hugo Zbinden e Nicolas Gisin, pela realização das medições em Genebra e discussões e empréstimo do contador de fótons.

Aos demais familiares e amigos que de uma forma ou de outra contribuíram para essa vitória.

A todos os professores e funcionários do CETUC pelo suporte acadêmico.

À Ericsson pelo suporte financeiro.

À VRAC e VRCOM da PUC-Rio pelo suporte financeiro. 


\section{Resumo}

de Faria, Giancarlo Vilela; von der Weid, Jean Pierre. Controle da polarização da luz em fibras ópticas monomodo e aplicações. Rio de Janeiro, 2009. 102p. Tese de Doutorado - Departamento de Engenharia Elétrica, Pontifícia Universidade Católica do Rio de Janeiro.

O controle de um estado de polarização, assim como o controle de todos os estados de polarização da luz transmitida através de uma fibra óptica monomodo, é realizado utilizando diferentes esquemas. O controle de polarização do sinal em um canal DWDM é realizado, utilizando sinais de referência em canais laterais em tempo contínuo. Uma troca de chave quântica codificada em polarização é discutida e realizada, no canal central, graças ao sistema de controle de polarização. A influência da distância espectral entre os canais e da PMD no controle também é estudada. Dois protótipos foram construídos, um sistema de controle de todos os estados de polarização e um compensador de PMD, e são apresentados e descritos inteiramente, incluindo os resultados dos seus testes.

O compensador de PMD foi testado, e medidas de compensação de PMD em um enlace de $43 \mathrm{~Gb} / \mathrm{s}$ foram realizadas em diversos formatos de modulação, bem como, em diversas situações de embaralhamento de polarização, com e sem o uso de FEC, a fim de avaliar a eficiência dos formatos de modulação, e a compensação de PMD com linhas de atraso de secção simples e múltiplas.

\section{Palavras-chave}

Polarização; Controle dos estados de polarização; Dispersão dos modos de polarização; Comunicações quânticas. 


\section{Abstract}

de Faria, Giancarlo Vilela; von der Weid, Jean Pierre (Advisor). Polarization of light control in monomode optical fibers and aplications. Rio de Janeiro, 2009. 102p. DSc. Thesis - Departamento de Engenharia Elétrica, Pontifícia Universidade Católica do Rio de Janeiro.

The control of polarization state, as well as a control of all states of polarization of a light signal travelling through a singlemode optical fiber, is performed using different schemes. The polarization control of the signal in a DWDM channel is performed using reference signals in lateral channels in real time. A polarization encoded quantum key distribution scheme is discussed and performed in the central channel through the control system polarization. The influence of the PMD and the spectral distance between channels in control is also investigated theoretically and experimentally. Two prototypes were built, a full polarization control system and a PMD compensator is shown and totally described, as well their test results. Measurements of PMD compensation on a link at $43 \mathrm{~Gb} / \mathrm{s}$ were performed in different modulation formats and in different situations of polarization scrambling, with and without the use of FEC in order to evaluate the efficiency of modulation formats, and PMD compensation with single or multiple section delay lines.

\section{Keywords}

Polarization; polarization states control; polarization mode dirpersion; quantum communications. 


\section{Sumário}

1 Introdução 19

2 Teoria 21

2.1. Polarização e representação Jones 21

2.2. Parâmetros de Stokes e Representação de Poincaré 24

2.3. Birrefringência e PMD 27

2.4. Qual a importância de se compensar a PMD? 30

2.5. O funcionamento básico do compensador óptico de PMD 32

2.6. A importância do sistema de controle de polarização nas comunicações quânticas $\quad 34$

2.7. Teoria do controle 36

3 O sistema de controle de polarização e o compensador de PMD 40

3.1. O controlador de polarização 40

3.2. A plataforma de controle 43

3.3. Os sinais de realimentação 44

3.4. Os drivers de tensão 45

3.5. Preparo do bit quântico 46

3.6. O algoritmo de controle 48

4 Resultados Experimentais em controle da polarização 52

4.1. Controle da birrefringência do canal quântico 52

4.2. Velocidade do sistema com controle de um SOP 54

4.3. Controle de todos os SOPs em um canal quântico por canais laterais $\quad 57$

4.4. Transmissão de bits quânticos codificados em polarização 63

4.5. Controle de todos os SOPs no canal central pela média dos SOPs dos canais vizinhos 
5 Resultados experimentais em compensação da PMD em 43Gb/s

5.1. Compensação da PMD pelo desvanecimento de um sinal de $40 \mathrm{GHz}$

5.2. O mapa de contorno da PMD

5.3. Montagem experimental para transmissão de $43 \mathrm{~Gb} / \mathrm{s}$

5.4. Embaralhamento da polarização

5.5. Medidas com formato NRZ:

5.6. Medidas no formato RZ-DQPSK:

82

5.7. Medidas no formato RZ-DPSK:

84

5.8. Comportamento do PMDC em presença do emulador de PMD estatístico em relação aos limites aceitáveis do FEC e OSNR.

5.9. Comportamento do PMDC em presença da DGD e embaralhador de polarização em relação aos limites aceitáveis do FEC e OSNR.

6 Conclusão 


\section{Lista de figuras}

Figura 1 - Onda plana se propagando na direção positiva do eixo $z$. Fonte: Keiser, G. "Optical Fiber Communications". McGrawHill, 2000.

Figura 2 - Ilustração das polarizações linear vertical, horizontal e a um ângulo $\theta$.

Figura 3 - Ilustração das polarizações circular à direita e circular à esquerda.

Figura 4 - Ponto P na esfera de Poincaré e suas coordenadas esféricas

$2 \chi$ e $\delta$

Figura 5 - Comportamento típico do pulso óptico em presença de uma fibra com PMD.

Figura 6 - Representação gráfica do vetor SOPMD e suas componentes.

Figura 7 - Medida típica da variação da DGD no Tempo e no comprimento de onda. Figura retirada de Adaptif_Component_Testing.pdf - Adaptif Photonics.

Figura 8 - Medidas de campo e as-built feitas em fibras instaladas 2005 mostram regularmente coeficientes de PMD de $0,05 \mathrm{ps} / \mathrm{km}^{1 / 2}$.

Figura 9 - Ilustração do realinhamento temporal entre as duas polarizações, resultando na compensação da PMD do enlace.

Figura 10 - Esquema de diferentes versões de linha de atraso na saída do controlador de polarização principal do compensador.

Figura 11 - Ilustração vetorial da compensação de PMD.

Figura 12 - Ilustração de um sinal com apenas um SOP e com dois SOPs não ortogonais.

Figura 13 - Ilustração da evolução da polarização na esfera em relação a variação da frequência óptica e da birrefringência com o tempo.

Figura 14 (a e b) - Direção do campo elétrico no guia em um 
controlador de polarização de LiNbO3.

Figura 15 - Representação dos eixos de rotação efetuados pelo controlador de polarização de LiNbO3.

Figura 16 - Diagrama da plataforma de controle baseada em FPGA.

Figura 17 - Diagrama de blocos simplificado do esquema de amplificação do sinal para acionamento do controlador de polarização.

Figura 19 - Ilustração das regiões de intensidades programadas para o algoritmo de histerese.

Figura 20 - Ilustração das regiões da esfera programadas para o algoritmo de histerese.

Figura 21 - Montagem para medição da velocidade de chaveamento de polarização.

Figura 22 - Pulso clássico no canal quântico com polarização determinada pelo bit de polarização.

Figura 23 - Pulso clássico no canal quântico com polarização determinada pelo bit de polarização.

Figura 24 - Evolução da polarização provocada por uma variação abrupta no controlador de polarização em $0,7 \mathrm{~s}$.

Figura 25 - Diagrama da montagem do experimento para a medição da velocidade de estabilização da polarização e da capacidade de estabilização em relação à velocidade de rotação da polarização.

Figura 26 - Tempo de resposta para a estabilização do sistema para o protótipo N.1.

Figura 27 - Tempo de resposta para a estabilização do sistema para o protótipo N.2.

Figura 28 - Gráfico da capacidade de estabilização traduzido pela potência transmitida em relação à frequência de rotação da polarização.

Figura 29 - Ilustração da montagem do sistema de controle de polarização aplicado a transmissão de bit quântico no regime de polarização.

Figura 30 (a e b) - Medidas da evolução da polarização na esfera de 
Poincaré com o sistema de controle de polarização desligado (a) e ligado (b).

Figura 31 - (a) Distribuição estatística do desvio angular na esfera de

Poincaré entre a polarização momentânea e a polarização desejada. (b) Distribuição estatística da perda de potência adicionada pelo sistema de controle de polarização devido ao defasamento entre o SOP controlado e o SOP alvo durante o experimento.

Figura 32 - Contagem de fótons para os estados de polarização fixos em Alice de $0^{\circ}$ e $45^{\circ}$.

Figura 33 - óptica adicionada ao longo de 10 horas de experimento.

Figura 34 - Diagrama do experimento de transmissão de bits quânticos codificados em polarização em um enlace com polarização estabilizada.

Figura 35 - Fidelidade entre os estados de polarização enviados por Alice e recebidos por Bob.

Figura 36 - Simulação da soma das projeções dos canais de referência alinhados a $45^{\circ}$ entre si.

Figura 37 - QBER óptica em relação a velocidade de rotação da polarização - Medição realizada por N. Walenta

Figura 38 - QBER do sistema de transmissão de qbits, (a) estabilização ativa e embaralhador desativo, (b) estabilização ativa e embaralhador ativo, (c) estabilização desativa e embaralhador ativo e (d) estabilizador reativado e embaralhador ativo Medições realizadas por N. Walenta.

Figura 39 - Diagrama do experimento de controle total da polarização do sinal em um canal central pelas médias dos SOPs de em canais vizinhos.

Figura 40 - Perda devido ao desalinhamento da polarização na transmissão de um sinal com polarização controlada com e sem a média dos SOPs dos sinais de canais vizinhos.

Figura 41 - Montagem do experimento para a verificação da flutuação do sinal de RF em função da PMD. 
Figura 42 - Montagem do experimento para a avaliação do compensador de PMD sob flutuação do sinal de RF puro em função da PMD.

Figura 43 - Intensidade do sinal de RF de $40 \mathrm{GHz}$ com o sistema compensado e não compensado em presença de um emulador de PMD de 12ps.

Figura 44 - Mapa de contorno da DGD x SOPMD onde as cores representam a função densidade de probabilidade conjunta para uma PMD média de 30ps.

Figura 45 - Ilustração da montagem do sistema de $43 \mathrm{~Gb} / \mathrm{s}$ para avaliação do compensador de PMD em presença de uma fonte programável de PMD de $1^{\mathrm{a}}$ e $2^{\mathrm{a}}$ ordem.

Figura 46 - Frequência máxima no espectro do sinal de realimentação versus o nível de velocidade do embaralhador de polarização.

Figura 47 - Mapa de contorno da ocorrência de rajadas de erro para o sistema de transmissão, no formato NRZ, não compensado.

Figura 48 - Mapa de contorno da ocorrência de rajadas de erro para o sistema de transmissão, no formato NRZ, compensado com linha de atraso de uma secção com 12ps.

Figura 49 - Mapa de contorno da ocorrência de rajadas de erros para o sistema de transmissão, no formato NRZ compensado com linha de atraso de duas secções $(6+9)$ ps.

Figura 50 - Mapa de contorno da ocorrência de rajadas de erros para o sistema de transmissão, no formato NRZ compensado com linha de atraso de duas secções $(9+6)$ ps.

Figura 51 - Mapa de contorno da ocorrência de rajadas de erros para o sistema de transmissão, no formato DQPSK com $2.0 \mathrm{~dB}$ de margem.

Figura 52 - Mapa de contorno da ocorrência de rajadas de erros para o sistema de transmissão, no formato DQPSK compensado com linha de atraso de duas secções $(6+4)$ os e $1.5 \mathrm{~dB}$ de magem.

Figura 53 - Mapa de contorno da ocorrência de rajadas de erros para o sistema de transmissão, no formato DPSK não compensado com 
Figura 54 - Mapa de contorno da ocorrência de rajadas de erros para o sistema de transmissão, no formato DPSK compensado com linha de atraso de duas secções $(6+4)$ ps e $2.5 \mathrm{~dB}$ de magem.

Figura 55 - Ilustração do esquema do emulador de PMD com misturadores de polarização.

Figura 56 - Ilustração da montagem do sistema de 43Gb/s para avaliação do compensador de PMD em presença de um emulador estatístico de PMD.

Figura 57 - Média da taxa de bits errados versus a magem da OSNR sem o uso do E.FEC.

Figura 58 - Frequência máxima de embaralhamento da polarização versus DGD

Figura A1 - Espectro de modulação dos códigos de linha em NRZ e

RZ.

Figura A1 - Representação do formato de modulação ASK.

Figura A2 - Representação do formato de modulação PSK.

Figura A3 - Representação do formato de modulação DPSK. 


\section{Lista de abreviaturas e Siglas}

A/D - Analog to digital (converter);

ASE - Amplified Spontaneous Emission;

AM - Amplitude modulation/modulator;

AT - Atenuador;

BB84 - Protocolo Bennet-Brassard 1984;

BER - Bit error rate;

D/A - Digital to analog (converter);

DEMUX - Demultiplexador;

DFB - Distributed feedback laser;

DGD - Differential Group Delay;

DOP - Degree of polarization;

DPSK - Differential Phase Shift Keying;

DQPSK - Differential Quadrature Phase Shift Keying;

DWDM - Dense Wavelength Domain Multiplexer;

E.FEC - Enhanced Forward Error Correction;

FBG - Fiber Bragg Grating;

FEC - Forward Error Correction;

FPGA - Field Programmable gate array;

MUX - Multiplexador;

NRZ - Non Return to Zero;

OSNR - Optical Signal to Noise Ratio;

PBS - Polarization Beamspliter;

PC - Polarization Controller;

PD - Photodetector;

PDCD - Polarization dependence chromatic dispersion;

PDG - Polarization Dependent Gain;

PDL - Polarization Dependent Loss;

PM - Phase Modulation/Modulator;

PMD - Polarization Mode Dispersion;

PMDC - Polarization Mode Dispersion Compensator; 
PMDS - Polarization Mode Dispersion Source;

PSP - Principal States of Polarization;

QBIT - Quantum Bit;

QKD - Quantum Key Distribution;

QBER - Quantum Bit Error Rate;

RZ - Return to Zero;

SOP - State of Polarization;

SOPMD - Second Order Polarization Mode Dispersion;

STM - Synchronous Transport Module. 